\title{
Sliding Spotlight SAR Processing for TerraSAR-X using a New Formulation of the Extended Chirp Scaling Algorithm
}

\author{
Josef Mittermayer*, Richard Lord** and Elke Börner*
}

\author{
*Deutsches Zentrum für Luft- und Raumfahrt (DLR) \\ 82234 Oberpfaffenhofen, Germany \\ T: +49-8153-28-2373, E-Mail: josef.mittermayer@dlr.de
}

\begin{abstract}
This paper describes the sliding spotlight algorithm and the processing strategy to be applied for TerraSAR-X. The steering spotlight geometry is analysed. Analysis of scene size and resolution demonstrates the particularities of this mode. The Doppler frequencies for individual targets and for a whole sliding spotlight scene are analyzed and the result shows the applicability of azimuth subaperture processing to sliding spotlight data. A description of the Extended Chirp Scaling Algorithm for sliding spotlight is presented.
\end{abstract}

Keywords: Sliding Spotlight, Extended Chirp Scaling, TerraSAR-X

\section{INTRODUCTION}

The German TerraSAR-X satellite [1] is implemented in Public-Private-Partnership between the German Aerospace Center (DLR) and the ASTRIUM GmbH. It is equipped with a high flexible phased array antenna allowing Stripmap, ScanSAR and Spotlight illumination. Two operational spotlight modes are planned, both in sliding geometry with 1 and $2 \mathrm{~m}$ azimuth resolution at 5 and $10 \mathrm{~km}$ along track scene extension, respectively [2].

The Extended Chirp Scaling Algorithm for Steering Spotlight [4] has been up-graded for the processing of TerraSAR-X sliding spotlight raw data. The up-grade consists more of a detailed analysis of the sliding spotlight geometry and raw data signal and doesn't mean the introduction of fundamental new processing steps. Also the way to analyse sliding spotlight geometry and raw data is similar to [4], w.r.t. scene and target illumination time, valid and illuminated target area, Doppler history of a complete scene of point targets, and division into subapertures. A comparison of this paper with [4] provides an understanding of the similarities and differences between steering and sliding mode.

\section{SLiding Spotlight GeOMETRY Definition}

The following assumptions and definitions are made:

1) the relative velocity $V_{\text {rel }}$ between the platform and the earth is used for the description of the geometry

2) a flat earth approximation [3] is introduced around the scene center for the maximum TerraSAR-X sliding spotlight scene, which has a maximum extension of $10 \mathrm{~km}$ in azimuth and ground range [2]. The approximation introduces an maximum error of $4 \mathrm{~m}$ in height between reference ellipsoid and flat scene and is thus well justified. [3]

3) a wide angle elevation antenna pattern compared to the cross-track scene extension is assumed

4) the squint angle is from antenna main to boresight direction With this assumptions, the geometry of a sliding spotlight mode is shown in figure 1 and is fully described by:

- illumination start and end time of the complete scene $t_{a, \text { start }}$ and $t_{a, \text { end }}$ (alternatively start and end squint angle $\psi_{\text {start }}$ and $\psi_{\text {end }}$ )

- antenna azimuth beamwidth $\theta_{\mathrm{a}}$

- azimuth and slant range position of rotation point $\left(t_{a}=0 ; r_{\text {rot }}\right)$

\author{
**University of Cape Town \\ Dept. of Electrical Engineering \\ 7700 Rodenbosch, Cape Town, South Africa
}

The rotation center is also situated on the approximation plane. The rotation center is only an auxiliary point, which is required for the calculation of several spotlight scene parameters like illumination times or antenna azimuth steering profile.

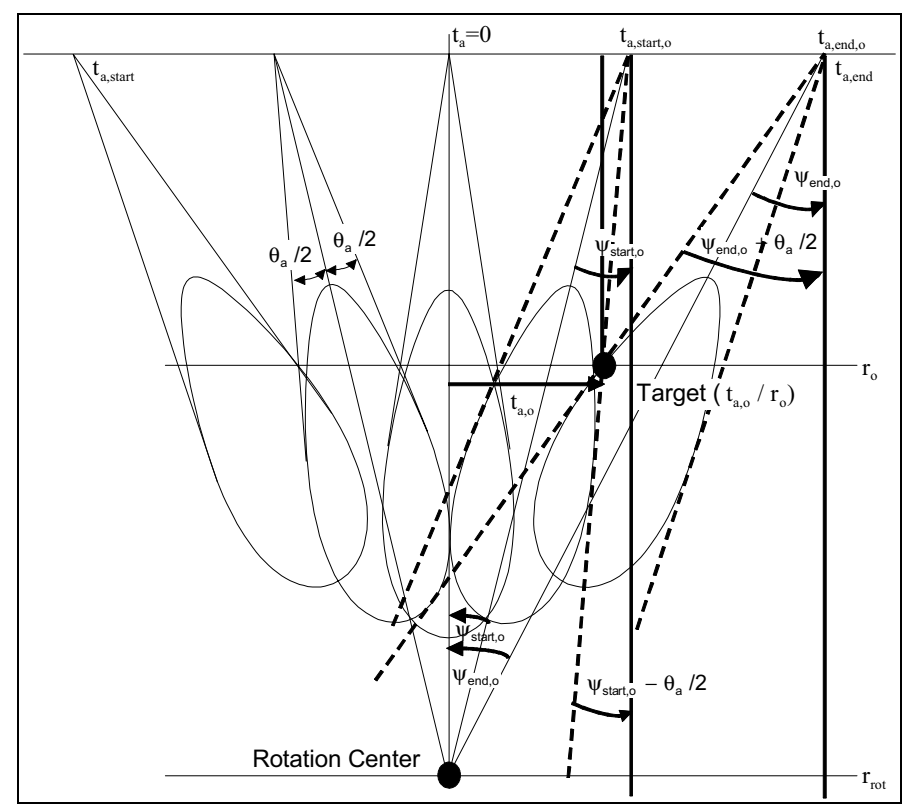

Figure 1. Sliding Spotlight Geometry Definition (exagerated steering angle)

A. Illumination Start and End Time of a Point Target

The illumination start and end of a point target is dependent on the point target position in azimuth and slant range $\left(\mathrm{t}_{\mathrm{a}, \mathrm{o}} ; \mathrm{r}_{\mathrm{o}}\right)$. From figure 1, the following two equations can be derived:

$$
\frac{\mathrm{V}_{\mathrm{rel}} \cdot \mathrm{t}_{\mathrm{a}, \text { end }, \mathrm{o}}}{\mathrm{r}_{\text {rot }}}=\tan \left(\Psi_{\text {end,o }}\right) ; \frac{\mathrm{V}_{\text {rel }} \cdot\left(\mathrm{t}_{\mathrm{a}, \text { end }, \mathrm{o}}-\mathrm{t}_{\mathrm{a}, \mathrm{o}}\right)}{\mathrm{r}_{\mathrm{o}}}=\tan \left(\Psi_{\text {end,o }}+\frac{\theta_{\mathrm{a}}}{2}\right)
$$

There are two equations for two variables and the combination of the two equations becomes:

$\tan ^{2}\left(\psi_{\text {end, },}\right)+\tan \left(\psi_{\text {end,o }}\right) \cdot\left[\frac{r_{o} / r_{\text {rot }}-1}{\tan \left(\theta_{a} / 2\right)}-\frac{t_{a, o} \cdot V_{\text {rel }}}{r_{\text {rot }}}\right]+\left[\frac{r_{o}}{r_{\text {rot }}}+\frac{V_{\text {rel }} \cdot t_{a, 0}}{r_{\text {rot }} \cdot \tan \left(\theta_{a} / 2\right)}\right]=0$

( 2 ) is first solved for $\Psi_{\text {end,o }}$, and then $t_{\mathrm{a} \text {,end,o }}$ is can be calculated:

$$
\begin{aligned}
& \tan \left(\Psi_{\text {end }, \mathrm{o}}\right)=\frac{\mathrm{V}_{\text {rel }} \cdot \mathrm{t}_{\mathrm{a}, \mathrm{o}}}{2 \cdot \mathrm{r}_{\text {rot }}}+\frac{1-\mathrm{r}_{\mathrm{o}} / \mathrm{r}_{\text {rot }}}{2 \cdot \tan \left(\theta_{\mathrm{a}} / 2\right)} \\
&(+) /-\sqrt{\left(\frac{\mathrm{V}_{\text {rel }} \cdot \mathrm{t}_{\mathrm{a}, \mathrm{o}}}{2 \cdot \mathrm{r}_{\text {rot }}}+\frac{1-\mathrm{r}_{\mathrm{o}} / \mathrm{r}_{\text {rot }}}{2 \cdot \tan \left(\theta_{\mathrm{a}} / 2\right)}\right)^{2}-\frac{\mathrm{r}_{\mathrm{o}}}{\mathrm{r}_{\text {rot }}}-\frac{\mathrm{V}_{\text {rel }} \cdot \mathrm{t}_{\mathrm{a}, \mathrm{o}}}{\mathrm{r}_{\text {rot }} \cdot \tan \left(\theta_{\mathrm{a}} / 2\right)}}
\end{aligned}
$$

The calculation of the start time $t_{a, \text { start,o }}$ starts analogously with:

$$
\frac{\mathrm{V}_{\text {rel }} \cdot \mathrm{t}_{\mathrm{a}, \text { start,o }}}{\mathrm{r}_{\text {rot }}}=\tan \left(\Psi_{\text {start,o }}\right) ; \frac{\mathrm{V}_{\text {rel }} \cdot\left(\mathrm{t}_{\mathrm{a}, \text { start,o }}-\mathrm{t}_{\mathrm{a}, \mathrm{o}}\right)}{\mathrm{r}_{\mathrm{o}}}=\tan \left(\Psi_{\text {start,o }}-\frac{\theta_{\mathrm{a}}}{2}\right)
$$

and the combination of the two equations results in: 


$$
\begin{aligned}
\tan \left(\Psi_{\text {start }, 0}\right) & =\frac{V_{\text {rel }} \cdot t_{a, 0}}{2 \cdot r_{\text {rot }}}+\frac{r_{o} / r_{\text {rot }}-1}{2 \cdot \tan \left(\theta_{a} / 2\right)} \\
+/(-) & \sqrt{\left(\frac{V_{\text {rel }} \cdot t_{a, 0}}{2 \cdot r_{\text {rot }}}+\frac{r_{o} / r_{\text {rot }}-1}{2 \cdot \tan \left(\theta_{a} / 2\right)}\right)^{2}-\frac{r_{0}}{r_{\text {rot }}}+\frac{V_{\text {rel }} \cdot t_{a, 0}}{r_{\text {rot }} \cdot \tan \left(\theta_{a} / 2\right)}}
\end{aligned}
$$

The illumination time of a target in sliding spotlight geometry $T_{\text {Slide }}\left(\mathrm{t}_{\mathrm{a}, \mathrm{o}} ; \mathrm{r}_{\mathrm{o}}\right)$ is dependent on target position $\left(\mathrm{t}_{\mathrm{a}, \mathrm{o}} ; \mathrm{r}_{\mathrm{o}}\right)$ and becomes:

$$
\mathrm{T}_{\text {Slide }}\left(\mathrm{t}_{\mathrm{a}, \mathrm{o}} ; \mathrm{r}_{\mathrm{o}}\right)=\mathrm{t}_{\mathrm{a}, \text { end }, \mathrm{o}}-\mathrm{t}_{\mathrm{a}, \text { start }, \mathrm{o}}=\frac{\mathrm{r}_{\text {rot }}}{\mathrm{V}_{\text {rel }}} \cdot\left(\tan \left(\Psi_{\text {end,o }}\right)-\tan \left(\Psi_{\text {start }, \mathrm{o}}\right)\right)
$$

\section{B. Valid and Illuminated Target Area Definition}

Figure 2 shows the valid target area in dark gray color and the illuminated target area being this area extended by the bright gray area. For all valid target positions applies:

- minimum and maximum processed target range $r_{o, m i n}$ and $r_{0, \max }$ are covered by the elevation pattern

- $r_{o, m i n}$ is resulting from the minimum required azimuth resolution, which becomes better in far range. Practically, $r_{o, m i n}$ is defined by the swath width for satellite geometry

- $r_{o, \max }$ is theoretically defined geometrically (see figure 2) and is always smaller than the rotation center, but practically $\mathrm{r}_{\mathrm{o} \text {,max }}$ is defined by the desired swath width

- the target illumination start time $t_{a, s t a r t, o}$ is greater or equal than the overall scene illumination start time $t_{a, s t a r t}$

- and the target illumination end time $t_{a, e n d, o}$ is smaller or equal than the overall scene illumination end time $t_{a, \text { end }}$

The minimum and maximum valid target azimuth position $t_{a, o, v a l i d, m i n}$ and $t_{a, o, v a l i d, m a x}$ at slant range $r_{o}$ is:

$$
\begin{aligned}
& \mathrm{t}_{\mathrm{a}, \mathrm{o}, \text { valid,min }}=\frac{\mathrm{r}_{\text {rot }}}{\mathrm{V}_{\text {rel }}} \tan \left(\Psi_{\text {start }}\right)-\frac{r_{\mathrm{o}}}{\mathrm{V}_{\text {rel }}} \cdot \tan \left(\Psi_{\text {start }}-\theta_{\mathrm{a}} / 2\right) \\
& \mathrm{t}_{\mathrm{a}, \mathrm{o}, \text { valid,max }}=\frac{\mathrm{r}_{\text {rot }}}{\mathrm{V}_{\text {rel }}} \tan \left(\Psi_{\text {end }}\right)-\frac{\mathrm{r}_{\mathrm{o}}}{\mathrm{V}_{\text {rel }}} \cdot \tan \left(\Psi_{\text {end }}+\theta_{\mathrm{a}} / 2\right)
\end{aligned}
$$

From $\mathrm{t}_{\mathrm{a}, \mathrm{o}, \mathrm{valid} \text {,min }}$ and $\mathrm{t}_{\mathrm{a}, \mathrm{o}, \mathrm{valid}, \max }$ the Sliding Spotlight Scene Size $\mathrm{X}_{\text {scene }}$ for a slant range $\mathrm{r}_{\mathrm{O}}$ results:

$X_{\text {scene }}=r_{\text {rot }} \cdot\left[\tan \left(\Psi_{\text {end }}\right)-\tan \left(\Psi_{\text {start }}\right)\right]-r_{0} \cdot\left[\tan \left(\Psi_{\text {end }}+\theta_{a} / 2\right)-\tan \left(\Psi_{\text {start }}-\theta_{a} / 2\right)\right]$

$\mathrm{X}_{\text {Scene }}$ is smaller in far range than in near range, in contrary to the steering spotlight mode [4].

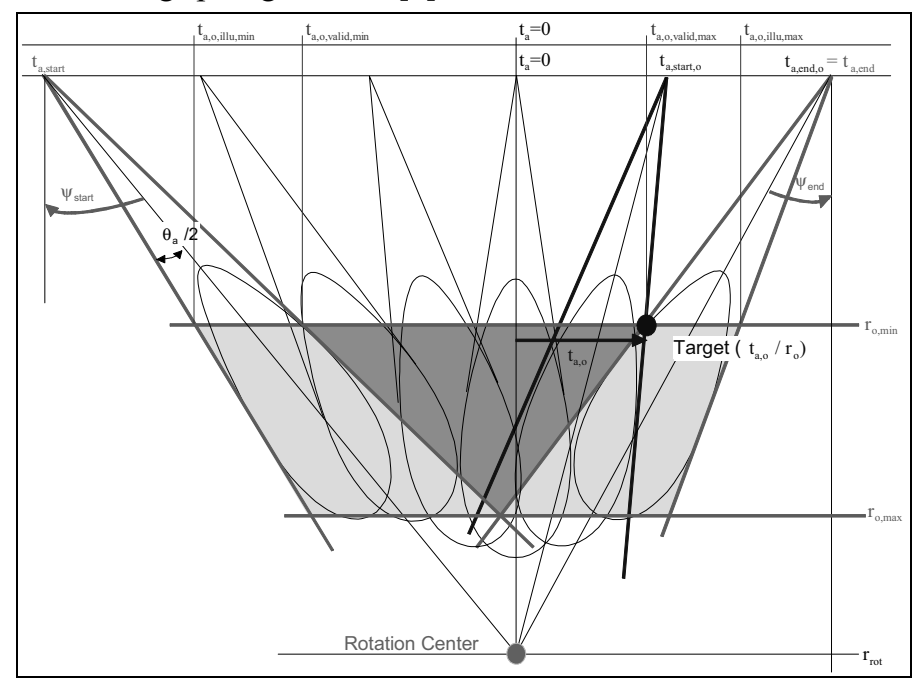

Figure 2. Valid and illuminated target area (exagerated steering angle)
The minimum and maximum illuminated azimuth position $t_{a, o, i l l u, m i n}$ and $t_{a, o, i l l u, m a x}$ of a fully or partially illuminated target at slant range $r_{0}$ is:

$$
\begin{aligned}
& \mathrm{t}_{\mathrm{a}, \mathrm{o}, \text { illu, min }}=\frac{\mathrm{r}_{\text {rot }}}{\mathrm{V}_{\text {rel }}} \tan \left(\Psi_{\text {start }}\right)-\frac{\mathrm{r}_{\mathrm{o}}}{\mathrm{V}_{\text {rel }}} \cdot \tan \left(\Psi_{\text {start }}+\theta_{\mathrm{a}} / 2\right) \\
& \mathrm{t}_{\mathrm{a}, \mathrm{o}, \mathrm{ill} \text {, max }}=\frac{\mathrm{r}_{\text {rot }}}{\mathrm{V}_{\text {rel }}} \tan \left(\Psi_{\text {end }}\right)-\frac{r_{\mathrm{o}}}{\mathrm{V}_{\text {rel }}} \cdot \tan \left(\Psi_{\text {end }}-\theta_{\mathrm{a}} / 2\right)
\end{aligned}
$$

\section{Azimuth Resolution}

The azimuth resolution is principally defined by the illumination length (or equivalently by the Doppler frequency variation) of a single target, i.e. $t_{a, \text { start,o }}$ and $t_{a, \text { end,o. The illumination }}$ start and end azimuth frequencies $\mathrm{f}_{\mathrm{a}, \text { start,o }}$ and $\mathrm{f}_{\mathrm{a} \text {,end,o }}$ for a point target at position $\left(t_{a, o} ; r_{0}\right)$ can be directly calculated from the instantaneous frequencies [4] at $t_{a, \text { start,o }}$ and $t_{a, e n d, o}$ :

$$
\begin{aligned}
\mathrm{f}_{\mathrm{a}, \text { start,o }} & =\frac{-2 \cdot \mathrm{V}_{\text {rel }}}{\lambda} \cdot \sin \left(\Psi_{\text {start,o }}-\frac{\theta_{\mathrm{a}}}{2}\right)=\frac{-2 \cdot \mathrm{V}_{\text {rel }}}{\lambda} \cdot \frac{\tan \left(\Psi_{\text {start,o}}-\frac{\theta_{\mathrm{a}}}{2}\right)}{\sqrt{1+\tan ^{2}\left(\Psi_{\text {start,o }}-\frac{\theta_{\mathrm{a}}}{2}\right)}} \\
& =\frac{-2 \cdot \mathrm{V}_{\text {rel }}^{2}}{\lambda} \cdot \frac{\mathrm{t}_{\mathrm{a}, \text { start,o }}-\mathrm{t}_{\mathrm{a}, \mathrm{o}}}{\sqrt{\mathrm{r}_{\mathrm{o}}^{2}+\mathrm{V}_{\text {rel }}^{2} \cdot\left(\mathrm{t}_{\mathrm{a}, \text { start,o }}-\mathrm{t}_{\mathrm{a}, \mathrm{o}}\right)^{2}}} \\
\mathrm{f}_{\mathrm{a}, \text { end }, \mathrm{o}} & =\frac{-2 \cdot \mathrm{V}_{\text {rel }}^{2}}{\lambda} \cdot \frac{\mathrm{t}_{\mathrm{a}, \text { end }, \mathrm{o}}-\mathrm{t}_{\mathrm{a}, \mathrm{o}}}{\sqrt{\mathrm{r}_{\mathrm{o}}^{2}+\mathrm{V}_{\text {rel }}^{2} \cdot\left(\mathrm{t}_{\mathrm{a}, \text { end }, \mathrm{o}}-\mathrm{t}_{\mathrm{a}, \mathrm{o}}\right)^{2}}}
\end{aligned}
$$

From $\mathrm{f}_{\mathrm{a} \text {,start,o }}$ and $\mathrm{f}_{\mathrm{a} \text {,end,o }}$, the azimuth resolution $\rho_{\mathrm{az}}\left(\mathrm{t}_{\mathrm{a}, \mathrm{o}} ; \mathrm{r}_{\mathrm{o}}\right)$ can be calculated for each target position:

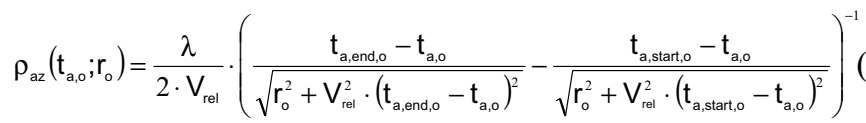

The azimuth resolution is better in far range than in near range since the illumination is longer in far range.

\section{SIMULATION WITH TERRASAR-X PARAMETER}

Using the formulas of the previous sections, the azimuth resolution and scene size was calculated for the example TerraSAR-X parameters of table 1.

\begin{tabular}{|l|l|}
\multicolumn{1}{c}{ TABLE I. } & TABLE TYPE STYLES \\
\hline scene center slant range & $837 \mathrm{~km}\left(55^{\circ}\right.$ inc. angle $)$ \\
\hline ground range scene extension & $10 \mathrm{~km}$ \\
\hline azimuth scene extension & $5 \mathrm{~km}$ \\
\hline azimuth resolution & $1 \mathrm{~m}$ \\
\hline rotation center slant range & $1330 \mathrm{~km}$ \\
\hline azimuth steering angle range & $1.141^{\circ}$ \\
\hline center squint angle & $0^{\circ}$ \\
\hline scene illumination time & $3.63 \mathrm{~s}$ \\
\hline
\end{tabular}

Figure 3 shows left the valid scene extension in azimuth

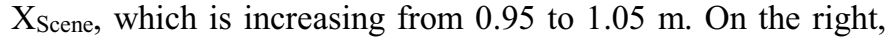
figure 3 shows the azimuth resolution for near (continuous line) and far range (dotted line) of the scene as a function of target azimuth position. The variation from near to far range is less than $1 \%$. There is a slight variation with azimuth which is too small to be seen in the plot. Please note that both plots correspond to processing exactly the $3 \mathrm{~dB}$ azimuth beamwidth, which is somehow arbitrarily and can be extended at cost of SNR. 


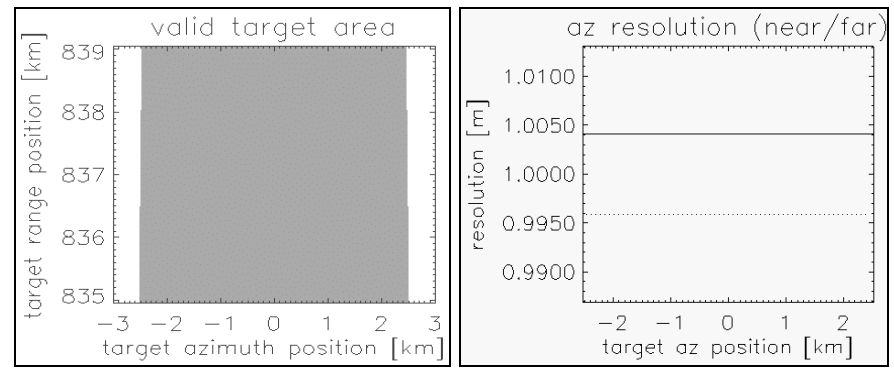

Figure 3. valid target area (left) and azimuth resolution (right) for example scene

\section{DOPPLER FREQUENCY ANALYSIS}

An analysis of the azimuth frequencies in sliding spotlight shows that the Doppler frequencies of all illuminated point targets, i.e. of the complete scene, are of course within the instantaneous bandwidth. This bandwidth is in-between min and max instantaneous azimuth frequency which are shown in figure 4 by the dotted lines and which are defined by the rotation center $r_{\text {rot }}$ and $\theta_{\mathrm{a}}$ :

$$
\begin{aligned}
& \mathrm{f}_{\mathrm{a}, \text { inst,max }}\left(\mathrm{t}_{\mathrm{a}}\right)=-\frac{2 \cdot \mathrm{V}}{\lambda} \cdot \sin \left(\operatorname{atan}\left(\frac{\mathrm{t}_{\mathrm{a}} \cdot \mathrm{V}}{\mathrm{r}_{\text {rot }}}\right)-\frac{\theta}{2}\right) \\
& \mathrm{f}_{\mathrm{a}, \text { inst,min }}\left(\mathrm{t}_{\mathrm{a}}\right)=-\frac{2 \cdot \mathrm{V}}{\lambda} \cdot \sin \left(\operatorname{atan}\left(\frac{\mathrm{t}_{\mathrm{a}} \cdot \mathrm{V}}{\mathrm{r}_{\text {rot }}}\right)+\frac{\theta}{2}\right)
\end{aligned}
$$

Figure 4 shows Doppler histories for several point targets at different slant range and azimuth positions, completely or only partially illuminated. Due to this, the subaperture strategy of steering spotlight [4] can be applied if the scene center slant range in the equations for the subaperture generation [4] is substituted by the rotation center slant range in the sliding spotlight mode. The sliding bandwidth mentioned in the figure provides the azimuth resolution.

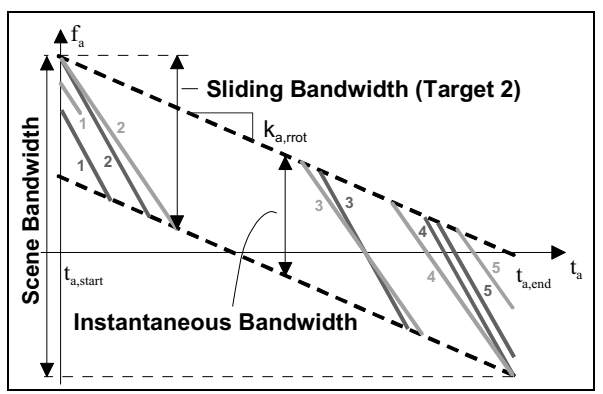

Figure 4. Sensor position at illumination of first and last valid target

\section{EXTENDED CHIRP SCALING FOR SLIDING SPOTLIGHT}

The basic structural formulation of the algorithm has been taken from [5]. The range compression is explained in detail in [6], while azimuth compression and subaperture method is accurately derived in [4]. Figure 5 gives an overview about the algorithm.

The raw data of a point target are shown in the upper plot on the left. The processing starts with an azimuth subaperture formation in order to avoid upsampling of the raw data in azimuth. The formation of the subapertures is indicated in the upper plot on the right by vertical lines. The subaperture approach allows a non-ambiguous azimuth frequency representation, the use of short azimuth FFT's, and the azimuth dependent update of processing parameters, e.g. the processing velocity.
Since in TerraSAR-X dechirp on receive is not applied, in the next steps, range cell migration correction and range compression is performed by chirp scaling. The result of the range processing is shown in the middle left plot. The signal is range compressed and without range cell migration.

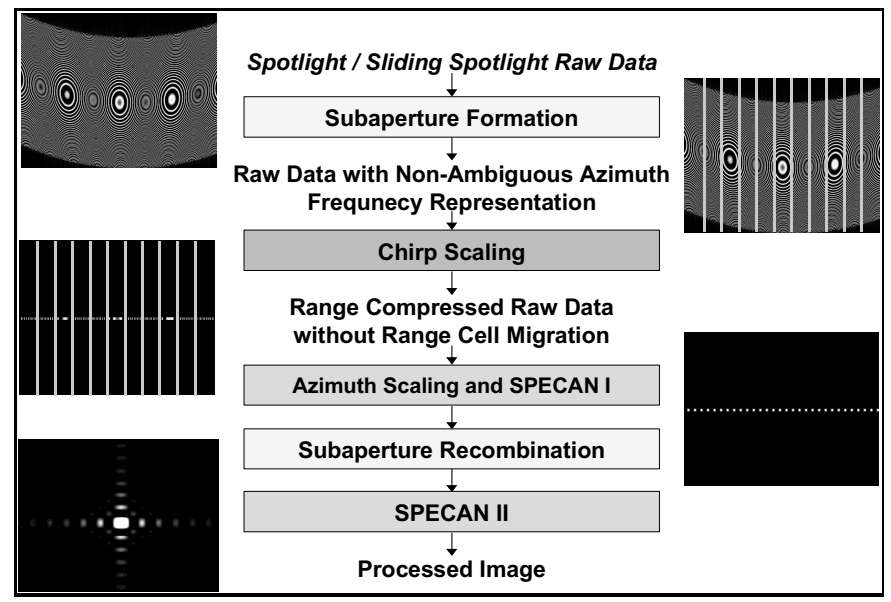

Figure 5. Extended Chirp Scaling for Sliding spotlight processing

The azimuth compression is performed by SPECtral ANalysis (SPECAN) combined with azimuth scaling. The two stages of the SPECAN processing are deramping (SPECAN I in figure 5) and final azimuth FFT (SPECAN II). The effect of the deramping operation on the point target signal can be seen in the lower plot on the right. The azimuth signal modulation is no more a linear frequency modulation but has changed to a constant frequency. This plot shows the signal after the recombination of the subapertures, i.e. there are no more vertical lines.

Finally, the last azimuth FFT (SPECAN II) compresses the signal in azimuth and the final result is the impulse response function in the lower left plot in the figure.

\section{DISCUSSION}

In a parallel paper of this conference [3], results of the processing of simulated TerraSAR-X raw data demonstrate the applicability of the algorithm for TerraSAR-X. It is shown, that the required processing accuracy is easily achieved and that the approximations inherent to a chirp scaling processing do not significantly influence the image quality.

\section{REFERENCES}

[1] M. Suess, S. Riegger, W. Pitz, R. Werninghaus: TERRASAR-X - Design and Performance, Proc. of EUSAR2002, Köln, Germany.

[2] J. Mittermayer, V. Alberga, S. Buckreuß, S. Riegger: TerraSAR-X: Predicted Performance, Proc. of Spie 2002.

[3] E. Börner, R. Lord, J. Mittermayer, R. Bamler: Evaluation of TerraSAR$\mathrm{X}$ Spotlight Processing Algorithm Accuracy based on a new Spotlight Raw Data Simulaor, Proc. IGARSS 2003.

[4] J. Mittermayer, A. Moreira, O. Loffeld: "Spotlight SAR Data Processing Using the Frequency Scaling Algorithm", IEEE Trans. on Geosc. and Remote Sensing, Vol. 37, No. 5, Sept. 1999, pp. 2198-2214.

[5] A. Moreira, J. Mittermayer and R. Scheiber: Extended Chirp Scaling SAR Data Processing in Stripmap, ScanSAR and Spotlight Imaging Modes, Proc. of EUSAR 2000.

[6] A. Moreira, J. Mittermayer and R. Scheiber, " Extended Chirp Scaling Algorithm for Air- and Spaceborne SAR Data Processing in Stripmap and ScanSAR Imaging Modes", IEEE Trans. on Geosci. and Remote Sensing, vol. 34, no. 5, pp. 1123-1136, September, 1996. 